\title{
Validation transculturelle d'une échelle de mesure des compétences sociales chez les élèves des 8 à 12 ans
}

\author{
Carine Simar(1), Julie Pironom(2), Damien Tessier(3), \\ Christelle Nsambu(4), Julien Masson(5)
}

(1) MCF en sciences de l'éducation, Université Clermont Auvergne, INSPÉ, ACTé

(2) Ingénieure d'études statisticienne, Université Clermont Auvergne, ACTé

(3) MCF en STAPS, UFRAPS, Université Grenoble-Alpes, Laboratoire SENS

(4) Étudiante en Master Santé Publique, Parcours "Éducation à la santé des enfants,

adolescents et jeunes adultes", Université Clermont Auvergne

(5) MCF en sciences de l'éducation, INSPÉ Académie de Lyon,

Lyon 1, Université Claude Bernard Lyon 1, Laboratoire HESPER

\begin{abstract}
Résumé : La notion de compétences psychosociale (CPS) a été introduite par l'OMS en 1993 et définie comme « la capacité d'une personne à répondre avec efficacité aux exigences et aux épreuves de la vie quotidienne. C'est la capacité d'une personne à maintenir un état de bien-être subjectif qui lui permet d'adopter un comportement approprié et positif à l'occasion d'interactions avec les autres, sa culture, son environnement. ». Aujourd'hui, les compétences psychosociales bénéficient de plus en plus de recherches et sont reconnues comme un déterminant solide du bien-être et donc plus largement de la santé des élèves. Si des programmes scolaires permettant de les développer voient le jour, il semblerait qu'il n'existe pas d'échelle psychométrique permettant de les mesurer. Cet article propose donc la validation d'une échelle de mesure de la compétence « sociale » des CPS, en langue française, pour un public âgé d'au moins 8 ans. Pour ce faire, un recueil auprès de 614 élèves de cycle 3 (CM1-CM2-6ème), âgés de 8 à 12 ans (moyenne $=9,71$ ans et écart-type $=0,81$ ) a été mené lors de l'année scolaire 2017-2018 dans le cadre d'un projet de recherche-intervention sur le développement des CPS. Les élèves ont complété un questionnaire auto-administré en classe créé à partir d'outils connexes existants. Après un traitement statistique (validité, fiabilité), il ressort que les propriétés psychométriques de l'échelle de mesure CPS relatives à la compétence « sociale»sont bonnes et permettent de la valider.
\end{abstract}

Mots-clés : Compétences psychosociales, école, échelle de mesure, compétence sociale.

\section{Introduction}

À l'heure actuelle, il est reconnu qu'agir en direction des jeunes constitue un des six domaines d'action prioritaires en matière de santé (Marmot et Bell, 2012). La revue d'articles publiés dans la série de The Lancet « Advancing Early Childhood Development : from Science to Scale » en 2016 dresse un constat assez préoccupant au niveau mondial : 43\% 
(249 millions) des enfants de moins de 5 ans dans les pays à revenu faible ou intermédiaire risquent de ne pas pouvoir se développer pleinement du fait de l'extrême pauvreté et d'un retard de croissance alors même que les interventions visant à développer les soins attentifs conjugués aux services existants permettraient d'améliorer la situation à faible coût.

En France, la situation est différente, mais reste préoccupante. Selon l'Organisation de Coopération et de Développement Économiques l'espérance de vie en France est relativement longue en raison notamment de la bonne qualité des soins délivrée par le système de santé, mais la réduction des facteurs de risque (e.g., inactivité physique, consommation nocive d'alcool, tabagisme) demeure un enjeu fort, en particulier chez les jeunes ${ }^{1}$. En effet, de nombreuses enquêtes européennes rapportent des constats alarmant concernant les facteurs de risques dans cette catégorie de la population. Par exemple, quatre enfants sur cinq ont un niveau d'activité physique insuffisant, en deçà des 60 minutes recommandées quotidiennement (Praznoczy et Larras, 2017). De plus, seuls $8 \%$ des jeunes de 17 ans n'ont jamais consommé d'alcool, de tabac ou de cannabis, et de nouveaux produits ou mode de consommation tels que la cigarette électronique, la chicha ou le narguilé apparaissent (Beck et al., 2016).

Afin d'endiguer cette tendance à l'accroissement des facteurs de risque, l'ensemble des actions visant les déterminants sociaux de la santé ou encore l'amélioration des conditions de vie quotidienne, figurent parmi les stratégies prometteuses pour diminuer les inégalités sociales de santé, favoriser le bien-être des populations et plus généralement promouvoir la santé. Plus précisément, l'un des leviers essentiels dans la promotion de la santé, notamment chez les jeunes est le développement des compétences psychosociales (CPS $)^{2}$. Cette stratégie promue au plan scientifique l'est également au plan politique dans beaucoup de contextes, notamment à l'école ${ }^{3}$. Toutefois, le développement d'outils de mesure valides des CPS constitue une des zones d'ombre importante de la littérature scientifique dans le champ de la promotion de la santé (Encinar et al., 2017). L'objectif de cet article est de présenter la première étape d'un travail consistant à développer un outil de mesure en langue française des compétences sociales chez l'enfant.

\section{Cadre théorique}

\subsection{Les compétences psychosociales}

Les compétences peuvent être considérées comme « la capacité d'exécution finalisée, liée à l'activité située d'un sujet et correspondant, chez lui, à une organisation d'éléments divers, notamment des savoirs et savoir-faire, ou d'unités cognitives construites, coordonnées, intégrées, structurées, hiérarchisées »(Chauvigné et Coulet, 2010). A l'heure actuelle, les CPS apparaissent dans la littérature scientifique comme une notion polysémique et polymorphe (Fonte et al., 2014), certains auteurs caractérisent les facteurs psychosociaux à un concept «fourre-tout » et « passe partout»(Saugeron et al., 2016).

1. OCDE (2016).

2. Organisation mondiale de la Santé (1993).

3. Ministère de l'éducation nationale (2020). 
Les compétences psychosociales peuvent être définies comme « des outils intellectuels et comportementaux qui permettent aux individus d'interagir de façon satisfaisante avec leurs environnements et d'exercer une influence positive sur eux-mêmes et leur entourage. Elles contribuent en ce sens à favoriser le bien-être physique, mental et social et à prévenir une large gamme de comportements et d'attitudes ayant des incidences négatives sur la santé des individus et des communautés, en particulier sur leur santé mentale » (Gorza et al., 2018). Ces compétences non académiques peuvent être définies aussi en référence à des indicateurs des capitaux culturel, social et émotionnel, capitaux sous-tendant celui du capital humain (Fanchini, 2016; Morlaix et Fanchini, 2018). Du point de vue de l'OMS (1993), la notion de CPS se définie comme « la capacité d'une personne à répondre avec efficacité aux exigences et aux épreuves de la vie quotidienne ». C'est la capacité d'une personne à maintenir un état de bien-être subjectif qui lui permet d'adopter un comportement approprié et positif à l'occasion d'interactions avec les autres, sa culture, son environnement (Luis et Lamboy, 2015).

En termes de classification des CPS, plusieurs catégorisations existent. Celle de l'OMS dénombre dix CPS qu'elle regroupe en cinq paires :

- Savoir résoudre les problèmes - savoir prendre des décisions.

- Avoir une pensée créatrice - avoir une pensée critique.

- Savoir communiquer efficacement - être habile dans les relations interpersonnelles.

- Avoir conscience de soi - avoir de l'empathie pour les autres.

- Savoir gérer son stress - savoir gérer ses émotions.

Ces dix compétences se répartissent, toujours selon l'OMS (1993) dans un cadre plus large constitué par trois grands domaines (Ndetei et al., 2018 ; World Health Organization, 1994, 2003, 2009) :

- Les compétences sociales (savoir communiquer, savoir résister à la pression des pairs, savoir faire preuve d'empathie, savoir collaborer, coopérer et être capable d'influencer positivement ses pairs)

- Les compétences cognitives (savoir prendre des décisions, résoudre des problèmes, avoir un esprit critique,)

- Les compétences émotionnelles (savoir faire face à ses émotions, gérer son stress, renforcer sa confiance en soi)

Les CPS sont largement reconnues comme un déterminant important de la santé et du bien-être, dans la mesure où elles constituent un facteur générique, c'est-à-dire associé à plusieurs problèmes de santé ${ }^{4}$. En effet, les pathologies et la mortalité évitables sont liés à une accumulation de comportements défavorables à la santé et à une insuffisance de comportements favorables. Or, ces comportements sont déterminés par un certain nombre de compétences et d'aptitudes, « médiateurs des comportements visés 》 (Mangrulkar et al., 2001). Ainsi, une action sur le renforcement de ces compétences permet d'induire une modification du comportement des individus, qui seront amenés à privilégier des choix

4. INSP Québec (2008). 
favorables à leur santé et à éviter les comportements défavorables. Actuellement, dans les démarches de prévention et de promotion de la santé, l'approche par le renforcement des CPS est souvent mise en avant, puisqu'elle permet de limiter les facteurs de risque et d'augmenter les facteurs de protection (Lamboy et Guillemont, 2014). A contrario, l'insuffisance du développement des CPS est l'un des déterminants majeurs de comportements à risque tels que la prise de substances psychoactives, les comportements violents et les comportements sexuels à risque, qui sont eux-mêmes des déterminants de pathologies (e.g., addictions, infections sexuellement transmissibles, troubles des conduites) (Luis et Lamboy, 2015). La littérature présente des résultats convergents quant à l'impact positif du développement des CPS sur l'amélioration du bien-être des jeunes et la réduction de leurs conduites à risques, et ce au travers d'études menées dans différentes pays : au Kenya (Ndetei et al., 2018), au Cambodge (Jegannathan et al., 2014), en Iran (Mohammadi, 2011), en Uganda (McMullen et McMullen, 2018), en Inde (Anand et al., 2015) ou bien encore en Allemagne (Menrath et al., 2015).

En dépit de ces résultats prometteurs, les études intervention sur les CPS se heurtent à deux limites majeures. La première est que la notion de CPS demeure assez floue sur le plan théorique. II n'existe pas de consensus dans la littérature permettant de circonscrire précisément les différentes composantes que recouvre cette notion. Cela induit la mise en œuvre d'intervention extrêmement hétérogènes pour développer les CPS. La seconde est qu'il n'existe actuellement aucune mesure permettant d'évaluer de manière précise et fiable les CPS des enfants (Wiglesworth et al., 2010). Cela limite la généralisation des résultats et empêche la comparaison des études entres elles. L'objet de la présente étude vise à apporter une contribution au dépassement de cette seconde limite.

Quatre éléments semblent participer du déficit actuel d'outils de mesure CPS validés scientifiquement en langue française adaptés aux jeunes en milieu scolaire : 1) l'acception même du terme CPS qui renvoie à un agrégat de dimensions et qui reste assez polysémique; 2) dans une grande majorité des publications, les CPS sont utilisées comme stratégie d'actions mais leur développement n'est pas mesuré, beaucoup d'auteurs se concentrent sur l'effet « protecteur » des CPS et leurs impacts sur les comportements à risques les indicateurs retenus sont ainsi liés aux comportements; 3) les CPS apparaissent avant tout comme un concept pragmatique; 4) l'introduction dans les politiques publiques des CPS en tant que stratégie explicite d'actions est assez récente.

\subsection{La mesure des compétences sociales}

Actuellement, les enquêtes nationales adressées aux adolescents en milieu scolaire (ex : HBSC, ESPAD, etc.) n'incluent pas de mesures spécifiques des CPS. En France, une revue de littérature a permis de repérer des outils d'évaluation dans la littérature professionnelle, mais ceux-ci n'ont pas fait l'objet d'une validation scientifique (Meuwly et al., 2011). Au niveau scientifique, la réflexion sur la mesure des CPS n'en est qu'au début, et il serait trop ambition de prétendre développer un outil global mesurant l'ensemble des dimensions. L'objet de cet article est de proposer une mesure de la compétence sociale des CPS pour des enfants de 8 à 12 ans.

L'analyse de la littérature internationale montre que la mesure des compétences sociales des enfants n'est pas une préoccupation récente, mais date de plus de quarante ans. Par 
exemple, l'étude de Johnson et Norem-Hebeisen (1979) visait à mesurer les dispositions à coopérer, à être dans la compétition ou être plus individuel chez 600 élèves de la maternelle au collège. Les résultats mettent en évidence les bonnes qualités psychométriques de l'outil au regard de ces trois dimensions. Toutefois, les compétences sociales sont ici définies en relation avec le travail scolaire, et non globalement dans les relations interpersonnelles comme les définit l'OMS. Plus récemment, Wills et al. (1996) ont développé une autre échelle destinée aux adolescents. II s'agit d'une échelle globale qui prend en compte différentes compétences émotionnelles (e.g., stress, état affectifs), cognitifs (e.g., processus d'adaptation) et sociales (e.g., soutien parental). La limite de cette échelle est qu'elle est spécifique à la consommation de produits stupéfiants, et que la dimension sociale se résume aux relations parents-adolescents. Dans une autre étude, Wang et al. (2008) ont examiné les qualités psychométriques du « Life Effectiveness Questionnaire Version H (LEQ-H) ». Adressé à 1264 élèves âgés de 12-14 ans, cette échelle est centrée sur l'évaluation des compétences de vie autour des dimensions suivantes : la gestion du temps, les compétences sociales, la motivation à la réussite, la tâche leadership, le contrôle émotionnel, participation et confiance en soi. Cette étude a révélé des propriétés satisfaisantes de cette échelle. Mais, au niveau des compétences sociales, sa pertinence semble limitée car elle ne mesure que des perceptions très générales (e.g., « je me sens en réussite dans mes relations sociales », « je communique bien avec les gens ») qui ne présente qu'un intérêt limité pour discriminer finement la maitrise des compétences sociales chez les enfants.

Dans le cadre d'une approche mixte de recherche (i.e., basée sur des méthodes qualitative et quantitative), Erawan (2010) a développé une échelle mesurant sept des dix compétences psychosociales définies par l'OMS (esprit critique, pensée créative, conscience de soi, empathie, relation interpersonnelle, prise de décision/résolution de problème et gérer ses émotions) plus une paire de compétences spécifiques au contexte thaïlandais (estime de soi / responsabilité sociale). Cette échelle, même si elle présente des caractéristiques psychométriques satisfaisantes s'adresse à un public lycée et se base sur des items peu adaptables à des enfants de 8-12 ans. Plus récemment, l'étude de Cronin et Allen (2017) conduite auprès de plus de 500 élèves (âgés de 10 à 21 ans) en section sportive s'est intéressée au développement d'une échelle de mesure afin d'évaluer dans quelle mesure ces étudiants perçoivent qu'ils développent leurs compétences psychosociales au travers de leur activité sportive. II ne s'agit à proprement parler des dix compétences psychosociales telles que définies par l'OMS mais de « life skills» regroupées sous huit compétences dans lesquelles on retrouve des points communs : le travail d'équipe, la capacité à se fixer des objectifs, la gestion du temps, les compétences émotionnelles, la communication interpersonnelle, les compétences sociales, le leadership, ainsi que la résolution de problèmes et la prise de décisions. Cette échelle demande ainsi aux sujets d'évaluer dans quel mesure le sport qu'il pratique leur a permis de renforcer les compétences précitées. Même si cette échelle possède des qualités psychométriques suffisantes (validité et fiabilité), les auteurs soulignent son caractère très contextuel. C'est une échelle validée et adaptée spécifique en référence à la pratique physique (Cronin et Allen, 2017).

Enfin, Weiss et al. (2014) ont développé un outil de mesure permettant d'évaluer chez les enfants - bénéficiant d'un programme de promotion des compétences psychosociales au travers de la pratique du golf - si les compétences acquises dans le cadre sportif sont 
transférables à d'autres domaines. S'agissant des compétences sociales, trois dimensions sont mesurées : la résolution des conflits, la capacité à demander l'aide des autres, et la capacité à aider les autres. Les résultats de cette étude révèlent les bonnes propriétés psychométriques de cet outil. D'autre part, cette étude montre que les enfants de 12 ans ayant suivis ce programme améliorent durablement leur CPS, et que ces dernières s'étendent à d'autres domaines que le sport, particulièrement dans le domaine des relations interpersonnelles.

En définitive, à l'issue de cette revue de littérature, l'échelle qui semble la plus adaptée aux enfants de 8 à 12 ans pour mesurer finement leurs compétences sociales est le « Life Skills Transfer Survey (LSTS) de Weiss et al. (2014). Ainsi l'objectif de cet article est de proposer la validation transculturelle des trois dimensions sociales de cet outil en examinant spécifiquement sa validité et sa fiabilité.

\section{Méthodologie de recherche}

\subsection{Participants et recueil}

Cette étude est la première étape d'un projet de recherche-intervention sur le développement des CPS, chez des élèves de cycle $3\left(\mathrm{CM} 1-\mathrm{CM} 2-6^{\mathrm{e}}\right)$, âgés de 8 à 12 ans. Six cent quatorze élèves issus de 14 établissements (treize écoles et un collège) de la région Rhône-Alpes $\left(54,0 \%\right.$ de garçons, $\mathrm{M}_{\mathrm{a} g e}=9,71$ ans, $\left.\mathrm{ET}=0,81\right)$ ont été impliqués dans cette étude. La répartition par classe s'établit ainsi : 45,6\% en CM1, 47,1\% en CM2 et $7,3 \%$ en $6^{\mathrm{e}}$. Les établissements sont répartis dans les départements de l'Ain, la Drôme, I'Isère et la Loire. Quatre sont dans des communes rurales (moins de 2000 habitants), trois dans des petites villes (2000 à 5000 habitants), quatre dans des villes moyennes (5000 à 10000 habitants) et trois dans des grandes villes (plus de 10000 habitants). Une deuxième mesure a été effectuée auprès de 564 élèves.

Un test-retest, espacé d'un mois à un mois et demi pour des raisons de faisabilité, a été réalisé sur un sous échantillon de 108 élèves issus de 4 établissements (58,3\% de garçons, $\mathrm{M}_{\text {âge }}=10,18$ ans, $\left.\mathrm{ET}=1,0\right)$ qui provenaient respectivement pour $38,9 \%, 30,6 \%$ et $30,6 \%$ de classe de CM1, CM2 et $6^{\mathrm{e}}$.

Les élèves ont complété un questionnaire auto-administré en classe, pour une durée allant de 42 minutes à $1 \mathrm{~h} 10$.

\subsection{Outils de mesure}

L'échelle sociale testée a été traduite et adaptée du Life Skills Transfer Survey (LSTS) (Weiss et al., 2014). Le LSTS se compose de huit dimensions (engager la conversation, réguler ses émotions, se fixer des buts, résoudre des conflits, faire des choix sains, apprécier la diversité, demander de l'aide, aider les autres) et comprend 50 items. La compétence sociale est mesurée à l'aide des trois dimensions « Résoudre des conflits 》 (8 items), «Demander de l'aide» (5 items) et « Aider les autres» (5 items).

Sur ces 18 items, quatre relatifs à la résolution des conflits avec les frères/sœurs ont été supprimé car l'objet est de valider un outil de mesure des compétences sociales dans le cadre scolaire. Comme dans la version originale du LSTS, les réponses sont portées sur 
une échelle en 5 points allant de $(1)$ « jamais » à $(5)$ 《 très souvent ». Le score à la compétence sociale est obtenu en calculant la moyenne des réponses aux item sur chaque dimension.

\subsection{Analyses des données}

L'analyse préalable des données a fait ressortir que les valeurs manquantes représentaient $1,5 \%$ des données et au maximum $3 \%$ au sein d'une même variable et qu'elles concernent $54,2 \%$ des élèves; les données sont manquantes aléatoirement. La potentielle perte de la moitié de l'échantillon fait apparaître la nécessité de remplacer les valeurs manquantes, la méthode d'imputation multiple de la chaîne de Markov de Monte Carlo (MCMC) (GlassonCicognani et Berchtod, 2010 ; Aurélien et Steve, 2016) a été utilisée en prenant en compte les réponses aux autres items de la même dimension.

La validité du construit a ensuite été examinée à l'aide d'une analyse factorielle exploratoire avec rotation Oblimin (AFE), puis d'une analyse factorielle confirmatoire (AFC). Pour cette dernière, l'ajustement du modèle aux données a été évalué par plusieurs indices d'adéquation : la statistique du $\chi^{2}$ d'ajustement, le Goodness of Fit Index (GFI), le Adjusted Goodness of Fit Index (AGFI), le Normed Fit Index (NFI), le Tucker Lewis Index (TLI), le Comparative Fit Index (CFI), le Root Mean Squared Error Of Approximation (RMSEA), l'Akaike Information Criterion (AIC), le Bayes Information Criterion (BIC). Selon les recommandations, des valeurs de GFI, AGFI, NFI, CFI et TLI supérieures à 0,95 ( $\mathrm{Hu}$ et Bentler, 1998), une valeur de RMSEA inférieure à 0,05 et un $\chi^{2}$ normé $\chi^{2} / \mathrm{ddl}$ inférieur à 3 (Roussel et al., 2005) représentent des indices d'ajustement d'un bon modèle.

La consistance interne a été testée en calculant les valeurs de l'alpha de Cronbach (Cronbach, 1951) et du rhô de Jöreskog (Jöreskog, 1971), moins sensible au nombre d'items de l'échelle. Une valeur supérieure à 0,7 est considérée comme satisfaisante, une valeur supérieure à 0,9 peut indiquer une redondance des items.

La fiabilité temporelle (i.e., entre le test et le retest) a été examinée grâce au calcul des kappa de concordance de Cohen et du coefficient de corrélation intraclasse entre les deux mesures ainsi que par la méthode de Bland et Altman (Berchtold, 2016 ; Elie et Colombet, 2011).

Afin de tester la validité concourante, des analyses de corrélation ont été réalisées avec d'autres mesures qui entretiennent des liens conceptuels avec les compétences psychosociales (Urdan et Schoenfelder, 2006; Williams et Galliher, 2006 ; Fortin, Plante et Bradley, 2011), et notamment : l'estime de soi scolaire, la satisfaction scolaire, la motivation, et les satisfactions des besoins de compétence, d'autonomie, et de proximité sociale.

Les données utilisées pour ces analyses ont été recueillies lors du temps 1 . Celles du deuxième recueil ont été utilisées, pour tester si le modèle établi par l'AFC n'était pas spécifique à notre premier jeu de données. Les analyses ont été réalisées avec SPSS 23 (descriptif, AFE, fiabilité, corrélations, comparaisons), AMOS 21 (AFC) et Jamovi 0.95.12 (méthode de Bland et Altman). Un seuil de significativité de $5 \%$ a été retenu. 


\section{Résultats}

\subsection{Acceptabilité et discrimination}

L'acceptabilité du questionnaire est bonne, le nombre de réponse manquante varie de 8 (soit $1,3 \%)$ à $15(2,4 \%)$ pour les items de la compétence sociale.

Aucun item ne présente d'effet plancher ou plafond. Au maximum, une modalité ne regroupe pas plus de la moitié des réponses sauf l'item 3 de la dimension « Demander de l'aide » où $62,4 \%$ des élèves ont choisi la dernière modalité.

\subsection{Reproductibilité}

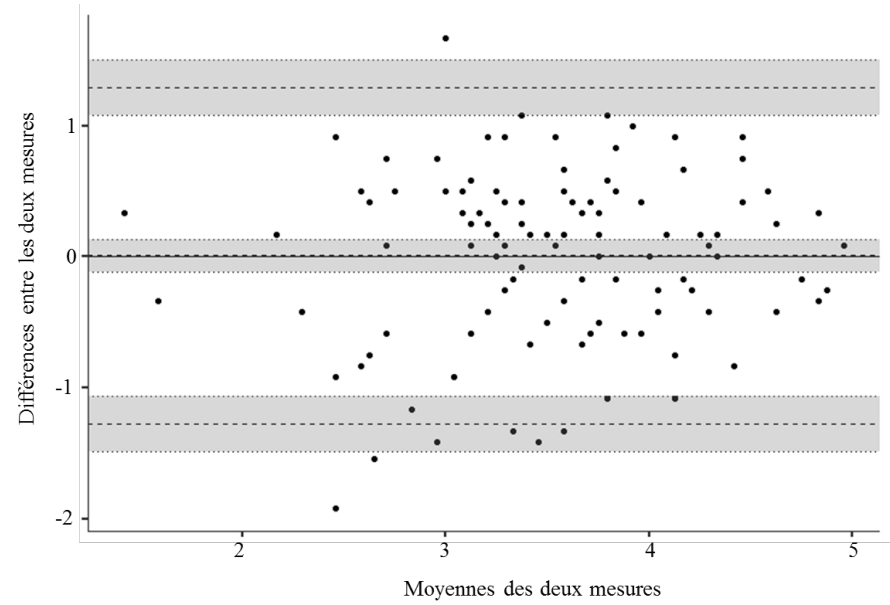

Figure 1 - Évaluation de la concordance avec le graphique de Bland-Altman pour la compétence sociale. Les points représente les couples de mesures du test-retest via leur moyenne et leur différence, les lignes sous forme de tirets sont la moyenne (au centre) et l'intervalle de confiance à $95 \%$, ils forment la zone de concordance. Les zones grisées sont les intervalles de confiance de ces valeurs.

Les mesures effectuées lors du test-retest montrent une concordance faible par item (les kappa sont compris entre 0,2 et 0,4$)$. Cependant, le coefficient de corrélation intra-classe pour le score représentant la compétence sociale indique une bonne corrélation entre les deux mesures $(\rho=0,77 ; \mathrm{p}<0,001)$.

Le graphique de Bland-Altman [fig. 1] permet d'appréhender la concordance entre les mesures. Il indique que la différence entre les deux mesures n'est pas dépendante ni proportionnelle de la valeur des mesures et que l'ensemble des points (couples de mesure) sauf trois se situent dans la zone de concordance.

\subsection{Analyse de la validité de construit}

\subsubsection{Analyse factorielle exploratoire}

Dans l'AFE, le critère de Kaiser suggère de retenir trois composantes ( $K M O=0,836$ ), expliquant $56 \%$ de la variance. La structure proposée est conforme à la structure théorique 
avec trois dimensions. Chaque item contribue à une et une seule dimension sauf l'item 5 de «Aider» qui a un poids factoriel inférieur à 0,4 sur toutes les composantes [tab. 1].

Le premier facteur (33\% de la variance) correspond à la dimension « Résoudre les conflits »(CONFLIT) avec des poids respectifs compris entre 0,53 et 0,81 . Le deuxième facteur $(13 \%)$ correspond à la dimension «Demander de l'aide » (DM-AIDE) avec des poids compris entre $-0,68$ et $-0,81$. Enfin le troisième facteur $(10 \%)$ est celui de la dimension «Aider les autres »(AIDE) avec des poids d'item se situant entre $-0,67$ et $-0,77$, celui de l'item 5 étant plus faible $(-0,31)$.

Tableau 1 - Poids factoriels de l'analyse factorielle exploratoire de la compétence sociale (rotation Oblimin).

\begin{tabular}{|l|c|c|c|}
\hline & Dimension 1 & Dimension 2 & Dimension 3 \\
\hline CONFLIT2 &, 808 &,- 028 &, 038 \\
\hline CONFLIT4 &, 802 &,- 046 &, 010 \\
\hline CONFLIT3 &, 714 &,- 049 &,- 003 \\
\hline CONFLIT1 &, 539 &, 054 &,- 109 \\
\hline DM-AIDE4 &,- 002 &,- 810 &,- 011 \\
\hline DM-AIDE3 &, 037 &,- 786 &,- 004 \\
\hline DM-AIDE2 &, 096 &,- 740 &, 049 \\
\hline DM-AIDE1 &, 070 &,- 701 &, 093 \\
\hline DM-AIDE5 &,- 213 &,- 683 &,- 255 \\
\hline AIDE1 &, 005 &, 013 &,- 766 \\
\hline AIDE3 &,- 048 &,- 013 &,- 732 \\
\hline AIDE2 &, 035 &, 041 &,- 729 \\
\hline AIDE4 &, 093 &,- 078 &,- 672 \\
\hline AIDE5 &, 263 &,- 065 &,- 306 \\
\hline
\end{tabular}

À l'exception du dernier item, l'analyse conforte la structure théorique.

\subsubsection{Analyse factorielle confirmatoire}

Le premier modèle testé dans l'AFC est celui qui correspond à sa définition théorique avec 14 items. Bien qu'il ait des qualités d'ajustement satisfaisantes (modèle 1 [tab2]), les indices de modifications fournies indiquent de fortes covariances entre les $5^{\text {es }}$ items des dimensions « Demander de l'aide » et «Aider les autres» et d'autres items/dimensions. Ces deux items ont été supprimés, les qualités d'ajustement du modèle ont été améliorées (modèle 2) [tab. 2]. Les indices GFI, AGFI, NFI, TLI et CFI sont supérieurs au seuil de 0,95 , le $\chi^{2}$ normé vaut 1,65 et le RMSEA est égal à 0,03 . La figure [fig2] représente ce dernier modèle avec les poids associés à chaque variable. 


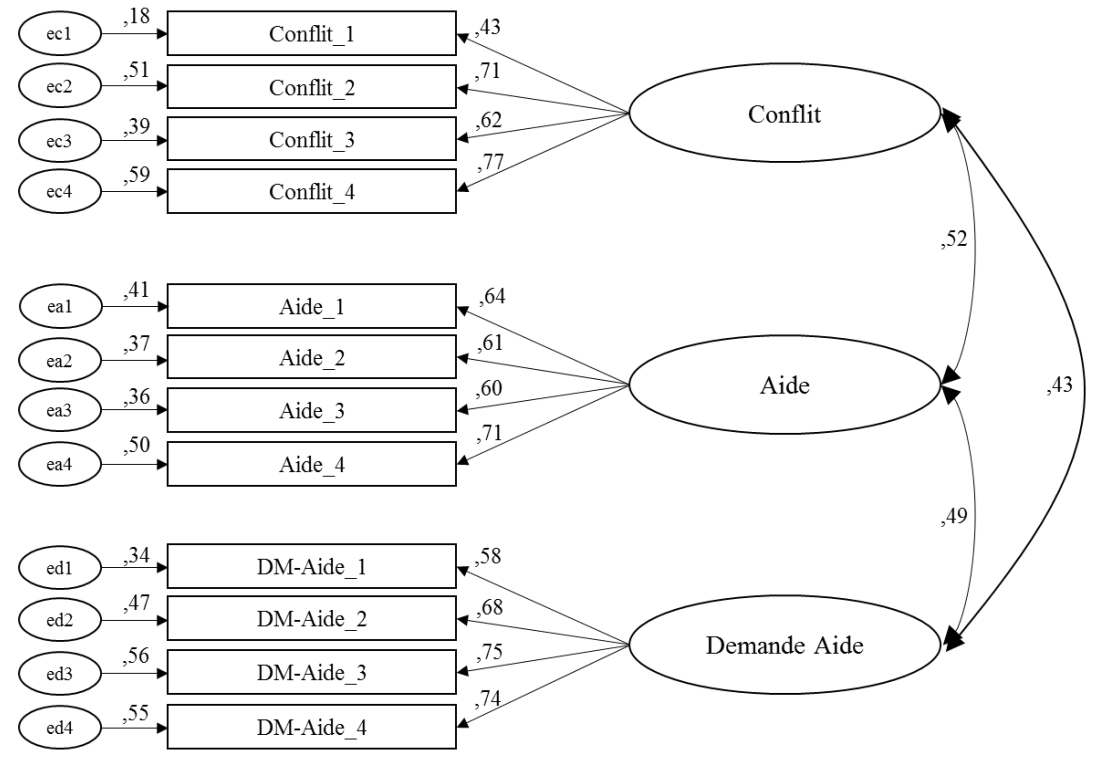

Figure 2 - Modèle 2 de l'AFC (12 items), les poids factoriels standardisés sont indiqués au-dessus des flèches.

Afin de s'assurer que le modèle ainsi établi n'est pas spécifique aux données du premier recueil, la structure en 12 items a également fait l'objet d'une AFC avec les données du temps de recueil 2 [tab2].

Tableau 2 - Qualité d'ajustement des modèles d'analyse factorielle confirmatoire de la compétence sociale aux temps 1 et 2 .

\begin{tabular}{|l|c|c|c|c|}
\hline \multirow{2}{*}{ Indices } & \multirow{2}{*}{ Seuils retenus } & \multicolumn{2}{|c|}{ Temps 1 } & Temps 2 \\
\cline { 3 - 5 } & & Modèle 1 & Modèle 2 & Modèle 2 \\
\hline Chi-2 (CMIN) & & 146,448 & 84,12 & 59,923 \\
\hline DDL & & 74 & 51 & 51 \\
\hline P(Chi-2) & & $<0,001$ & $<0,001$ & $<0,001$ \\
\hline GFI & $>0,90$ & 0,967 & 0,978 & 0,974 \\
\hline AGFI & $>0,90$ & 0,953 & 0,966 & 0,960 \\
\hline RMSEA & $<0,05$ & 0,040 & 0,033 & 0,022 \\
\hline NFI & $>0,95$ & 0,938 & 0,957 & 0,963 \\
\hline TLI & $>0,95$ & 0,960 & 0,977 & 0,993 \\
\hline CFI & $>0,95$ & 0,968 & 0,982 & 0,994 \\
\hline Chi-2 / DDL & Plus faible & 1,979 & 1,649 & 1,175 \\
\hline AIC & Plus faible & 208,448 & 138,12 & 113,923 \\
\hline BIC & Plus faible & 345,214 & 257,239 & 218,998 \\
\hline
\end{tabular}


Les qualités d'ajustement sont très satisfaisantes, et même supérieures à celles obtenues pour le temps 1 .

\subsection{Analyse de la fiabilité par la consistance interne}

Les alpha de Cronbach valent 0,73 pour les dimensions « Résoudre les conflits » et «Aider les autres » et 0,78 pour «Demander de l'aide ». Les valeurs des rhô de Jöreskog prennent respectivement comme valeurs $0,79,0,80$ et 0,80 [tab3]. Aucune suppression d'item ne permet d'améliorer la valeur des alpha de Cronbach.

Tableau 3 - Consistance interne des dimensions (alpha de Cronbach et rhô de Jöreskog)

\begin{tabular}{|l|c|c|c|}
\hline Dimensions & Nombre d'items & Alpha de Cronbach & Rhô de Jöreskog \\
\hline Seuil recherché & & Entre 0,7 et 0,9 & \\
\hline Conflit & 4 & 0,725 & 0,793 \\
\hline Demande d'aide & 4 & 0,782 & 0,798 \\
\hline Aide & 4 & 0,733 & 0,799 \\
\hline
\end{tabular}

Ces valeurs, toutes comprises entre 0,7 et 0,9 , indiquent une bonne consistance interne sans redondance des items.

\subsection{Validité concourante}

Les corrélations entre la compétence sociale et les autres mesures externes aux CPS dans le questionnaire ont été calculées afin d'établir la validité concourante [tab4].

Tableau 4 - Validité concourante, corrélations existant entre la compétence sociale et les variables dépendantes

\begin{tabular}{|l|c|c|}
\hline $\begin{array}{l}\text { Corrélations avec la } \\
\text { compétence sociale }\end{array}$ & $\begin{array}{c}\text { Coefficient de } \\
\text { corrélation } \rho\end{array}$ & $\mathrm{p}$ \\
\hline Estime de soi & 0,104 & 0,010 \\
\hline Satisfaction scolaire & 0,306 & $<0,001$ \\
\hline Motivation intrinsèque & 0,323 & $<0,001$ \\
\hline Motivation identifiée & 0,307 & $<0,001$ \\
\hline Motivation contrainte & 0,029 & 0,478 \\
\hline Compétence & 0,307 & $<0,001$ \\
\hline Autonomie & 0,141 & $<0,001$ \\
\hline Proximité sociale & 0,303 & $<0,001$ \\
\hline
\end{tabular}

Toutes les corrélations obtenues sont significatives hormis la motivation contrainte. Les corrélations avec les échelles d'estime de soi et d'autonomie sont plutôt faible $(0,10<$ $\rho<0,15)$ et moyennes pour les autres $(0,30<\rho<0,33)$. 


\section{Discussion}

À l'heure actuelle, force est de constater que de nombreux programmes et projets en matière de CPS (Unplugged, GBG, etc.) se développent en direction de publics variés (jeunes, familles, etc.), c'est le cas également auprès de publics atteints de maladies chroniques notamment dans le cadre de l'ETP. En effet, les CPS sont reconnues comme facteur de protection en matière de santé afin d'améliorer le bien-être ou bien encore prévenir des conduites à risques. Bien que cette approche soit promue, le concept de CPS reste encore peu étayé théoriquement dans certaines pratiques professionnelles ou projets. Pour autant, à l'heure actuelle, les auteurs s'accordent à dire que la classification en trois compétences (sociales, émotionnelles et cognitives) est reconnue comme pertinente sur le plan théorique.

L'objectif de cet article était de proposer la validation transculturelle de la dimension sociale du LSTS (Weiss et al.,2014) reposant sur trois dimensions : la résolution de conflits, la demande d'aide et la proposition d'aide. Cette échelle de mesure des compétences sociales présente les caractéristiques suivantes : elle repose sur une structure théorique à trois dimensions et s'adresse à un public âgé à minima de 8 ans.

Le développement de ces compétences sociales (aussi appelées compétences de vie) ont fait l'objet de multiples travaux notamment dans le sport (Chinkov et Holt, 2016 ; Goudas, 2010). En outre, l'évaluation des programmes tels que «The first Tee » a permis de démontrer à travers une étude longitudinale de 3 ans son efficacité sur le développement des compétences sociales (Weiss et al., 2016). Afin d'évaluer ces compétences, le choix a été fait de mobiliser les travaux relatifs à l'échelle de mesure « Life Skills Transfer Survey » (LSTS) - adaptés en langue française. En effet, celle-ci a fait l'objet des validations suivantes : validité structurelle, validité convergente et consistance interne (Weiss et al., 2014).

Les résultats de l'étude démontrent que le questionnaire est bien accepté par les élèves et montre une bonne discrimination par les items. La concordance par item apparait comme faible, ce qui peut être dû à l'écart entre les deux passations du test-retest qui est élevé (supérieur à un mois), mais elle ne remet pas en cause la concordance sur l'ensemble de la compétence. II ressort des différentes analyses relatives à la validité de construit que la définition théorique retenue est bonne, en effet l'AFE conforte la structure théorique (hormis l'item 5) ainsi que l'AFC conduite au cours des trois années (suppression de deux items). Concernant l'analyse de la fiabilité, les tests conduits pour mesurer la consistance interne (alpha de Cronbach et Rhô de Jöreskog) sont satisfaisants de même que les analyses de corrélation (test-restest) indiquant une bonne fiabilité de mesure. Enfin, concernant la validité concourante, il ressort des analyses que les corrélations sont significatives (hormis la motivation contrainte). En bref, il ressort de cet article que les propriétés psychométriques (validité et fiabilité) de l'échelle de mesure CPS relatives aux compétences « sociales» sont bonnes et permettent de la valider.

\section{Conclusion}

En conclusion, cette étude poursuivait comme objectif principal la validation psychométrique des compétences «sociales»de l'échelle de mesure CPS à partir de l'adaptation 
transculturelle du questionnaire LSTS. Les résultats sont en faveur de la validation scientifique de ces compétences de l'échelle de mesure des CPS en langue française et adaptée aux jeunes âgés d'au moins 8 ans, ce qui vient compléter les construits déjà validés ou en cours de validation (compétences émotionnelles et cognitives).

Ces premiers résultats font émerger de nouveaux questionnements scientifiques. Un premier enjeu a trait au suivi du développement des CPS des jeunes afin d'affiner la compréhension que nous avons actuellement des modalités et des caractéristiques de développement de ces compétences dans une perspective longitudinale. Un autre questionnement est lié à l'étude des facteurs intervenant sur le développement des CPS des jeunes et à la mesure de leurs impacts. Le dernier enjeu a trait, selon nous, à la nécessaire extension en matière de validation scientifique d'échelles de mesure des CPS à des publics présentant des caractéristiques différentes compte tenu des politiques publiques développées en ce sens.

\section{Références bibliographiques}

Anand, T., Ingle, G. K., Meena, G. S., Kishore, J. et Yadav, S. (2015). Effect of life skills training on dietary behavior of school adolescents in Delhi : A nonrandomized interventional study. Asia-Pacific Journal of Public Health, 27(2), NP1616-1626.

Aurélien, N. K. A. et Steve, K. K. (2016). Gestion des données manquantes dans les bases de donnees en sciences sociales : algorithme NIPALS ou imputation multiple? European Scientific Journal, ESJ, 12(35), 390.

Beck, F., Adès, J-E., Lermenier-Jeannet, A., Cadet-Taïrou, A., Le Nézet, O., Mutatayi, C., Ngantcha, M. et Obradovic, I. (2016). Jeunes et addictions, éléments de synthèse. Paris : OFDT.

Berchtold, A. (2016). Test-retest : Agreement or reliability? Methodological Innovations, 9, 2059799116672875. https://doi.org/10.1177/2059799116672875\url\{https://doi.org/10.1177/2059799116672875\}

Chauvigné, C. et Coulet, J.-C. (2010). L'approche par compétences : Un nouveau paradigme pour la pédagogie universitaire? Revue française de pédagogie. Recherches en éducation, 172, 15-28.

Chinkov, A. E. et Holt, N. L. (2016). Implicit transfer of life skills through participation in Brazilian jiu-jitsu. Journal of applied sport psychology, 28(2), 139-153.

Cronbach, L. J. (1951). Coefficient alpha and the internal structure of tests. Psychometrika, 16(3), 297-334.

Cronin, L. D. et Allen, J. (2017). Development and initial validation of the Life Skills Scale for Sport. Psychology of Sport and Exercise, 28, 105-119.

Elie, C. et Colombet, I. (2011). Methods of evaluation of reproducibility. Sang Thrombose Vaisseaux, 23(3), $138-145$.

Encinar, P.-E., Tessier, D. et Shankland, R. (2017). Compétences psychosociales et bien-être scolaire chez l'enfant : Une validation française pilote. Enfance, 1, 37-60.

Erawan, P. (2010). Developing life skills scale for high school students through mixed methods research. European Journal of Scientific Research, 47(2), 169-186.

Fanchini, A. (2016). Les compétences sociales et la réussite scolaire des élèves de cycle III : l'effet de l'accompagnement scolaire. Thèse de doctorat non publiée, Université de Bourgogne Franche-Comté, Dijon.

Fonte, D., Apostolidis, T. et Lagouanelle-Simeoni, M-C. (2014). Compétences psychosociales et éducation thérapeutique du patient diabétique de type 1 : Une revue de littérature. Sante Publique, 26(6), 763-777.

Fortin, L., Plante, A. et Bradley, M. F. (2011). Recension des écrits sur la relation enseignant-élève. Chaire de recherche de la Commission scolaire de la Région-de-Sherbrooke sur la réussite et la persévérance scolaire. [En ligne] : https://www.csrs.qc.ca/fileadmin/user_upload/Page_Accueil/Enseignants/Fenetre_pedagogique/PEPS

/Relation-maitre-eleve.pdf

Glasson-Cicognani, M. et Berchtold, A. (2010). Imputation des données manquantes : Comparaison de différentes approches. Communication présentée aux 42èmes Journées de Statistique, Marseille, France, mai. 
Gorza, M., Houzelle, N., Sempé, S., Lasbeur, L., Hamsany, M., Roscoät, E. D. et Cardoso, T. (2018). Promouvoir la santé de l'enfant en soutenant les compétences parentales : L'action de Santé publique France. Enfances Psy, $77(1), 37-48$.

Goudas, M. (2010). Prologue : A review of life skills teaching in sport and physical education. Hellenic Journal of Psychology, 7(3), 241-258.

Hu, L. et Bentler, P. M. (1998). Fit indices in covariance structure modeling : Sensitivity to underparameterized model misspecification. Psychological Methods, 3(4), 424-453.

Jegannathan, B., Dahlblom, K. et Kullgren, G. (2014). Outcome of a school-based intervention to promote life-skills among young people in Cambodia. Asian Journal of Psychiatry, 9, 78-84.

Johnson, D. W. et Norem-Hebeisen, A. A. (1979). A Measure of Cooperative, Competitive, and Individualistic Attitudes. The Journal of Social Psychology, 109(2), 253-261.

Jöreskog, K. G. (1971). Simultaneous factor analysis in several populations. Psychometrika, 36(4), 409-426.

Lamboy, B. et Guillemont, J. (2014). Développer les compétences psychosociales des enfants et des parents : Pourquoi et comment? Devenir, 26(4), 307.

Luis, É. et Lamboy, B. (2015). Les compétences psychosociales : Définition et état des connaissances. La Santé en Action, 431, 12-16.

Mangrulkar, L., Whitman, C. et Posner, M. (2001). Life skills approach to child and adolescent healthy human development. Washington : Pan American Health Organization.

Marmot, M. et Bell, R. (2012). Fair Society, Healthy Lives. Public health, 126(1), S4-S10.

McMullen, J. D. et McMullen, N. (2018). Evaluation of a teacher-led, life-skills intervention for secondary school students in Uganda. Social Science \& Medicine, 217, 10-17.

Menrath, I., Prüßmann, M., Müller-Godeffroy, E., Prüßmann, C., Ottova, V., Ravens-Sieberer, U. et Thyen, U. (2015). Effectiveness of School-Based Life Skills Programmes on Secondary Schoolchildren in a High Risk Sample. Gesundheitswesen (Bundesverband Der Arzte Des Offentlichen Gesundheitsdienstes (Germany)), 77(1), S76-77.

Meuwly, M., Bray, M. et Bollier, A. (2011). Comment travailler avec les enfants et leur environnement. Manuel de compétences psychosociales. Lausanne : Terre des hommes.

Mohammadi, A. (2011). Survey the Effects of Life Skills Training on Tabriz High School Student's Satisfaction of Life. Procedia - Social and Behavioral Sciences, 30, 1843-1845.

Morlaix, S. et Fanchini, A. (2018). Le développement du capital humain en lien avec les compétences psychosociales. Communication présentée au 7ème colloque international d'UNIRéS Symposium «, Éducation à la santé et compétences psychosociales ». Paris, France, octobre.

Ndetei, D. M., Mutiso, V., Gitonga, I., Agudile, E., Tele, A., Birech, L., Musyimi, C. et McKenzie, K. (2018). World Health Organization life-skills training is efficacious in reducing youth self-report scores in primary school going children in Kenya. Early Intervention in Psychiatry, 13(5), 1146-1154.

Praznoczy, C. et Larras, B. (2017). État des lieux de l'activité physique et de la sédentarité en France - Mise à jour de l'édition 2017. Clermont-Ferrand: Observatoire national de l'activité physique et de la sédentarité. http://www. onaps.fr/data/documents/Onaps_TAB2017_Mise-a-jour.pdf

Roussel, P. (2005). Chapitre 9. Méthodes de développement d'échelles pour questionnaires d'enquête. Dans P. Roussel et Wacheux, F. (Eds.), Méthodes \& Recherches. Management des ressources humaines (pp. 245-276). Bruxelles : De Boeck Supérieur.

Saugeron, B., Sonnier, P. et Marchais, S. (2016). COMETE : Un outil pour développer les compétences psychosociales en éducation thérapeutique du patient. Santé Publique, 28(2), 157-161.

Urdan, T. et Schoenfelder, E. (2006). Classroom effects on student motivation : Goal structures, social relationships, and competence beliefs. Journal of school psychology, 44(5), 331-349.

Wang, C.-K., Neill, J., Liu, W.C., Tan, O.-S., Koh, C. et Ee, J. (2008). Project work and life skills : Psychometric properties of the life effectiveness questionnaire for project work. Educational Research Journal, 23(1), 21-43.

Weiss, M.R., Bolter, N.D. et Kipp, L.E. (2014). Assessing Impact of Physical Activity-Based Youth Development Programs : Validation of the Life Skills Transfer Survey (LSTS). Research Quarterly for Exercise and Sport, 85(3), 263-278.

Weiss, M. R., Bolter, N. D. et Kipp, L. E. (2016). Evaluation of The First Tee in promoting positive youth development : Group comparisons and longitudinal trends. Research quarterly for exercise and sport, 87(3), $271-283$. 
Wigelsworth, M., Humphrey, N., Kalambouka, A. et Lendrum, A. (2010). A review of key issues in the measurement of children's social and emotional skills. Educational Psychology in Practice, 26(2), 173-186.

Williams, K.L. et Galliher, R.V. (2006). Predicting Depression and Self-Esteem from Social Connectedness, Support, and Competence. Journal of Social and Clinical Psychology, 25(8), 855-874.

Wills, T.A., Vaccaro, D., McNamara, G. et Hirky, A.E. (1996). Escalated substance use : A longitudinal grouping analysis from early to middle adolescence. Journal of Abnormal Psychology, 105(2), 166-180.

\section{Références institutionnelles}

Institut national de santé publique du Québec, Desjardins, N. et Laverdure, J. (2008). Avis scientifique sur les interventions efficaces en promotion de la santé mentale et en prévention des troubles mentaux : présenté au Ministère de la santé et des services sociaux. Développement des individus et des communautés, Institut national de santé publique : Québec.

Ministère de l'éducation nationale. (2020). VADEMECUM : l'école promotrice de santé. EduSanté. [En ligne] : https://cache.media.eduscol.education.fr/file/Sante/84/2/VM_EcolePromotriceSante_1240842.pdf

OCDE. (2016). Comment la France se positionne ? Panorama de la santé : Europe 2016. State of health in the EU cycle. [En ligne] : http://www.oecd.org/fr/sante/systemes-sante/Health-at-a-Glance-EUROPE-2016-Note -FRANCE.pdf. (03/05/2020)

Organisation Mondiale de la Santé. (1993). Life Skills Education in schools.

World Health Organization life-skills training is efficacious in reducing youth self-report scores in primary school going children in Kenya. Early Intervention in Psychiatry, 13(5), 1146-1154.

World Health Organization. (1994). Life Skills Education for Children and Adolescents in schools. Introduction and Guidelines to Facilitate the Development and Implementation of Life Skills Programmes. Programme on Mental Health [En ligne] : https ://apps.who.int/iris/bitstream/handle/10665/63552/WHO_MNH_PSF_93.7ARev.2.pdf ?sequence $=1$ \&isAllowed $=y(03-05-2020)$.

World Health Organization. (2003). Skills for health : skills-based health education including life skills : an important component of a Child-Friendly/Health-Promoting School [en ligne] https://apps.who.int/iris/handle/10665/ 42818 (03-05-2020).

World Health Organization. (2009). Preventing violence by developing life skills in children and adolescents [en ligne] https://apps.who.int/iris/handle/10665/44089 (03-05-2020). 